\title{
Special Issue on Healthcare Mechatronics
}

\author{
Yan Shi ${ }^{1 *}$, Qing Guo ${ }^{2}$ and Bin Zhang ${ }^{3}$
}

Healthcare mechatronics is a typical multidisciplinary field involving machinery, medicine, computer, and automation, which has been widely applied in respiratory therapy, urology robot, rehabilitation exoskeleton, artificial heart, etc. Existing progresses has some defects in modeling, design and implementation of healthcare mechatronics. Therefore, exploring new design theories, key technologies and typical applications is an effective to promote the rapid development of this field.

We received 9 papers in this special issue and there are 4 papers selected for publication, covering several aspects such as blood cooling system, serial urology manipulator, lower limb exoskeleton driven by hydraulic and motor actuators.

Firstly, a locally mixed sub-low temperature device proposed by Wang et al. is used to simulate the human blood transfer process via the cold and hot water mixing experiment. Then the static characteristics of the heat transfer system are described by the mathematic model construction and the experimental station verification. By using the parametric regulation, the results of simulation experiments show that $14.52{ }^{\circ} \mathrm{C}$ refrigeration can reduce the original temperature of $33.42{ }^{\circ} \mathrm{C}$ to $32.02{ }^{\circ} \mathrm{C}$, and the temperature of refrigerated blood rises to $18.64{ }^{\circ} \mathrm{C}$, and the average error is about $0.3{ }^{\circ} \mathrm{C}$. Meanwhile, the efficiency of the heat exchange system also increases significantly, as the thermal conductivity of the vascular sheath increases. Furthermore, the mass increases and the temperature of the mixed blood temperature decreases, as the input cold blood flow rate increases. This work develops subsequent research on local fixed-point sub-low temperature control technology.

\footnotetext{
*Correspondence: yesoyou@gmail.com

1 School of Automation Science and Electrical Engineering, Beihang

University, Beijing 100191, China

Full list of author information is available at the end of the article
}

Secondly, a urology robot is used in the minimally invasive surgery of prostatic hyperplasia and tumor. The distribution error of the fixed point is presented by Shi et al. Meanwhile, the tracking accuracy of manipulator end effector on the cone bottom surface of the workspace is guaranteed by the designed control algorithm of the manipulator. By using the digital twin technique, the trajectory mapping and synchronization control are realized between virtual model and the actual manipulator. The experimental results show that compared with PD feedback control, the feed-forward controller can achieve a reduction of $30.0 \%$ in the average error of the fixed point of the manipulator and a reduction of $33.3 \%$ in the maximum error.

Then a novel hydraulic actuating system (HAS) based on electric-hydrostatic actuator is proposed by Sun et al. which is applied to hip and knee joints to improve the robot's overloading performance. Each HAS includes an electric servo motor, a high-speed micro pump, a specific tank, and other components into a module. Based on the human motion data and load requirements, the dynamic models of the HAS are constructed to validate the system identification. The trajectory tracking and humanexoskeleton interaction experiments have verified the satisfactory compensation effect of external load. Compared with the previous prototype, the total weight of the HAS is reduced by about $40 \%$, and the power density is increased by 1.6 times.

Finally, a humanoid prototype of 2-DOF lower limb exoskeleton is developed by Chen et al. to evaluate the wearable comfortable effect between person and exoskeleton. To improve the measurement accuracy of the human-robot interaction torque, the BPNN is adopted to estimate the interaction force and to compensate for the measurement error of the 3D-force/torque sensor. Meanwhile, the backstepping controller is designed to realize the exoskeleton's passive position control, which means that the person passively adapts to the exoskeleton. On 
the other hand, a variable admittance controller is used to implement the exoskeleton's active follow-up control, which means that the person's motion is motivated by his/her intention and the exoskeleton control tries best to improve the human-robot wearable comfortable performance. To improve the wearable comfortable effect, serval regular gait tasks with different admittance parameters and step frequencies are statistically performed to obtain the optimal admittance control parameters. The BPNN compensation algorithm and two controllers are verified by the experimental exoskeleton prototype with human-robot cooperative motion.

We are fully aware of the on-going research and development efforts on healthcare mechatronics academic field and industry. It is hoped that the selected papers of the special issue can provide some foresight for future research and development, and a good reference for industry practices. Furthermore, we also hope that these work can drive more and more research results about healthcare mechatronics by medical-industrial field intersection and integration in depth. In the future, enough academic research and production applications in healthcare mechatronics will promote the development of our country's intelligent medical device field.

\section{Acknowledgements}

Not applicable.

\section{Authors' Information}

Yan Shi is a full professor at School of Automation Science and Electrical Engineering, Beihang University, China. He received his doctoral degree in mechanical engineering from Beihang University, China. Dr. Shi received the Top Young Talents of National Ten Thousand Talents Plan in 2018, and won the Second Prize of National Science and Technology Progress Award in 2017. From 2013 to 2019, he won three times of the First and Second Prize of Machinery Industry Federation. His research interests include mechatronic engineering, intelligent medical devices and energy saving technologies of pneumatic systems.
Qing Guo, received his B.E. degree in automation from Harbin Institute of Technology, China, in 2003, and M.S. and Ph.D. degrees in navigation, guidance and control from Harbin Institute of Technology, China, in 2005 and 2008, respectively.

Now Dr. Guo is a full professor at School of Aeronautics and Astronautics, University of Electronic Science and Technology of China. Dr. Guo is also the backup candidates for Academic and Technical Leaders in Sichuan Province, IEEE senior member, member of Fluid Transmission and Control Branch, Chinese Mechanical Engineering Society, senior member of Chinese Mechanical Engineering Society, Youth Expert Group Leader of the Committee on Fluid Control and Engineering, Chinese Mechanical Engineering Society. From

December 2013 to December 2014, he was an academic visitor with Center for Power Transmission and Motion Control, Department of Mechanical Engineering, University of Bath, UK. Dr. Guo has more than 50 publication journal and conference papers, the academic editor of Hydraulic and Pneumatic. His research interests include robust and adaptive control, electrohydraulic, exoskeleton \& rehabilitation robot.

Bin Zhang, is an associate researcher and a doctoral supervisor at State Key Lab of Fluid Power \& Mechatronic Systems, School of Mechanical Engineering, Zhejiang University, China. He was a visiting scholar at Institute of Biomedical Engineering, University of Oxford. Dr. Zhang is the director of Joint International Research Lab on 3D Bioprinting of Zhejiang University-University of Oxford, associate editor of Bio-Design and Manufacturing, senior member of Chinese Mechanical Engineering Society.

\section{Funding}

Not applicable.

\section{Competing Interests}

The authors declare no competing financial interests.

\section{Author Details}

${ }^{1}$ School of Automation Science and Electrical Engineering, Beihang University, Beijing 100191, China. ${ }^{2}$ School of Aeronautics and Astronautics, University of Electronic Science and Technology of China, Chengdu 611731, China.

${ }^{3}$ The State Key Laboratory of Fluid Power and Mechatronic Systems, Zhejiang University, Hangzhou 310027, China.

Received: 9 January 2021 Accepted: 19 January 2021

Published online: 01 February 2021

\section{Submit your manuscript to a SpringerOpen ${ }^{\odot}$ journal and benefit from:}

- Convenient online submission

- Rigorous peer review

- Open access: articles freely available online

- High visibility within the field

- Retaining the copyright to your article

Submit your next manuscript at springeropen.com 